of opinion that there is always an hysterical basis and that the evidences of toxæmia are the consequence of dehydration. He supports his view by striking instances of severe cases relieved by pure suggestion; and although there has been opposition from those who accept the traditional toxæmic hypothesis, I think he has gained adherents among practitioners with considerable obstetric experience.

(2) Reflex Causes.-The vomiting centre may be excited by stimuli from the stomach itself and by afferent fibres from other parts of the body via the vagus. A convenient classification of all these causes will be: $(a)$ Gastric, including emetics, improper food, excess of ordinary food, irritant poisons, pyloric stenosis including the so-called congenital hypertrophic stenosis of infants, dilatation of the stomach, ulcer and malignant disease of the stomach, venous congestion as in morbus cordis and hepatic cirrhosis.

(b) General visceral causes. Appendicitis and peritonitis generally. Intestinal obstruction. Dietl's crises. Pelvic disease-possibly pregnancy may be included in this category. Pulmonary phthisis. Violent coughing from any cause. Shock from a blow in the epigastrium or on the testicle or other particularly sensitive surface.

(c) Through the central nervous system. (1) The special senses-e.g., a revolting smell or taste, a repulsive or terrifying spectacle; (2) the brainconcussion, meningitis, hæmorrhage, tumour, migraine, mal de mer, hysteria. Perhaps the vomiting which is not uncommon in anæmia may be included here. (3) The spinal cord-gastric crises.

It is impossible to consider all these conditions in any detail, nor is such consideration really necessary. In many the cause is obvious, or vomiting is only an incident accompanied by other prominent symptoms. So that a careful general examination and regard to the history should prevent error, and it is rarely difficult to trace the cause as an essentially gastric one when the relation of the act to food and the character of the material vomited are remembered.

In most acute abdominal conditions vomiting is not the predominating symptom, but appears as the result of pain which occupies the forefront of the patient's sensations. The elementary precaution of observing the temperature is always advisable to eliminate the occurrence of acute illness.

In dealing with a chronic vomiting suspected to be of gastric origin it is well to have a radiographic examination in order to define without delay whether organic pyloric stenosis is present, a condition which may demand operation, whatever the cause of the stenosis.

\section{Hysterical Vomiting.}

Cerebral vomiting I have already considered, and a final word may be given to hysterical vomiting.
The type I have described above, the soldier with some sort of psychic war trauma, is nowadays very uncommon, but hysterical vomiting is probably older than warfare itself. It is most frequently encountered in young women and mistaken for gastric ulcer. It may be conceded that some difference of opinion exists as to the percentage of cases of genuine gastric ulcer who vomit, but $I$ think one is safe in saying that fully 50 per cent. of cases suffer in addition to those cases of pyloric stenosis which sometimes results from chronic ulceration. And whilst exceptions exist, in the large majority of cases the pain of ulcer is relieved by the act of vomiting. In hysterical vomiting, on the other hand, a not infrequent history is that vomiting does not relieve any pain which may be present. The patient's nutrition remains conspicuously good, and it is no uncommon feature to see her vomit in the course of a meal and return quite complacently to finish. It is a nasty blunder to condemn a genuine organic case as hysterical and the diagnosis should be most carefully considered, always including radiography; but once it is arrived at there should be no half-hearted attempt at treatment. Psychotherapists say that the condition is dependent upon a psychic cause for disgust usually of the nature of a sexual trauma the memory of which persists in the subconscious. I cannot speak of this with any experience, let alone authority, but I have certainly seen rapid cures by the ordinary psychotherapeutic measures of persuasion and suggestion. The injunction of a strong-minded sister to "stop vomiting and not be such a fool " may not commend itself as a scientific procedure in the eyes of the psychoanalyst, but in certain cases this simple psychotherapeutic method has sufficed to terminate abruptly a case of long duration.

\section{PYOGENIC INFECTIONS OF THE SKIN IN CHILDREN.*}

\author{
BY
}

A. M. H. GRAY, C.B.E., M.D., F.R.C.P. LoND., PHYSICIAN FOR DISEASES OF THE SKIN, HOSPITAL FOR SICK CHILDREN, GREAT ORMOND-STREET, LONDON,

INFECTIONS of the skin by streptococci and staphylococci are particularly common in infants and children, doubtless because the protective horny layer is less well developed than in older persons, and also because they are less careful of the hygiene of the skin and are more liable to come in close contact with infected persons than are adults.

It is possible to divide pyogenic infections into two classes: (1) those in which a pure primary

* Post-graduate Lesture delivered at the Hospital for Sicis Children, Great Ormond-street, on March 25th, 1926. 
infection of one or other of these organisms occurs, and (2) those in which the infections are mixed or are secondary contaminations of some preexisting non-pyogenic skin affection. The first group is more or less well-defined and will be dealt with in detail, but the second group is a much more complex one and can only be just touched upon in this lecture.

\section{STREPTOCOCCAL INFECTIONS.}

\section{Impetigo Contagiosa.}

This is the commonest of the streptococcal affections of the skin and is familiar, in its typical form, to you all. The primary lesion is a small, clear vesicle, varying in size from a pinhead to a pea, or sometimes larger, and occurring chiefly on the parts of the body uncovered by the clothes. It is chiefly seen on the face. The vesicle is very thin-walled, occurring immediately beneath the horny layer of the skin and above all the other epidermal layers. It is, therefore, very easily ruptured, and when ruptured the exudate from the underlying mucous layer dries up to form a crust. In the majority of cases vesicles are not seen when the case comes under observation, so that impetigo contagiosa is often looked upon purely as a crusted eruption. When the vesicle remains anruptured for a few hours, the contents become turbid from the presence of pus cells. If there are many pus cells in the exudate, the crust, when formed, takes on a dirty greenish colour; if, however, pus cells are absent, the crust is of transparent amber colour.

In simple cases the lesions are few in number and quite discrete. They usually spread from a single focus of infection and are, therefore, asymmetrical in distribution. In some cases, however, the lesions run together and great masses of crust are formed. Individual crusts are not as a rule surrounded by an inflammatory halo, the crust having the appearance of being stuck on normal skin.

Impetigo contagiosa is usually a direct infection from another infected individual, but sometimes is a secondary infection from ear discharge, nasal discharge, or from scratching, as in scabies and pediculosis. The distribution of the eruption will frequently suggest the source of the original infection; for example, an impetigo in the occipital region suggests always pediculosis capitis.

Sometimes we find that the original vesicles do not rupture, but continue to spread, so that large blisters or bullæ are formed. This occurs usually in the more virulent infections in bigger children, but is more or less the rule in infants, whose skin appears less resistant. This type of case is called bullous impetigo. Another form is also occasionally seen. The original vesicle spreads, but sinks down in the centre and dries there as a thin papery crust. The edge goes on spreading as it heals in the centre.
Eventually ringed lesions are formed, which unit with neighbouring ones, and thus a large number of circinate and gyrate figures may be formes occupying a considerable area of the face and spreading down on to the trunk. The eruption io often not unlike a seborrhœic dermatitis, but can: be distinguished by the narrow blister edge whic $\overrightarrow{F_{3}}$ is always present in this form, which we cait circinate impetigo. Although impetigo contagiose is thought to be generally due to streptococca䑧 infection, there is reason for believing that some cases are due to infection by pyogeniz staphylococci.

The treatment of this condition is usually simplee It consists in removing the crusts and applying $\vec{a}_{\vec{a}}$ mild antiseptic application. In mild cases crustes can be removed mechanically, by bathing witto weak lysol solution, or by soaking in warm olive oil. If they are very thick and adherent, they are. best removed by starch poultices. The most popular antiseptic ointment is dilute white precipitate ointment. Personally, I think this is a very messfe preparation, as it melts and runs all over the faco and does not remain on the lesions. I prefer to use Lassar's paste, to which 3 per cent. ammoniated or yellow oxide of mercury has been added. Thi sticks well to small lesions and can be spread on muslin and tied on to larger lesions. Healing is usually rapid under such an application. It is suitable in the ordinary and in the circinate typeso It is not so suitable, however, for the bullous lesions. In these cases lotions are more suitable and I find 1 in 1000 flavine solution one o the best. The blisters should be punctured? the escaping fluid carefully mopped up, and th $\vec{E}$ lotion applied on linen and changed every few hours.

Some writers state that it is advisable to give vaccines, even in the early cases of impetigo. The are often given in the more persistent cases, bu I believe that if proper attention is given to local treatment this is not generally necessary.

\section{Pemphigus Neonatorum.}

This is merely a form of bullous impetigo occurring in newly born infants. Infection usuall takes place from the mother or midwife. Cases vary much in severity, some running a very rapi\$ and fatal course. Some very severe cases rapidlfs strip the horny layer off the whole surface of the body and are spoken of as Ritter's disease oण exfoliative dermatitis of infants. They occueㅏㄹ luckily very rarely, in institutions and are probably due to a specially virulent organism? Pemphigus neonatorum is a very infectious conv dition, and if a case occurs in a lying-in or children' $\bar{\theta}$ hospital, the most stringent precautions arg necessary to prevent spread.

Like impetigo contagiosa, the lesions spread fron one localised spot and are not symmetrical. Af first the infant may be in good health, only when the eruption becomes extensive does the genera 
condition suffer. Cases must not be confused with congenital syphilitic pemphigus, which is a symmetrical eruption coming out chiefly on the palms, soles, and buttocks, and is associated with other types of syphilitic rashes and general signs such as wasting and snuffles.

The treatment of pemphigus neonatorum is on the same lines as for bullous impetigo, but care must be taken not to apply lotions to large areas of the body, as they cause great loss of heat. I generally use 1 per cent. ammoniated mercury in calamine liniment applied on strips of lint to small areas at a time, each area as treated being covered by a layer of cotton-wool.

\section{Flexural Impetigo.}

Impetigo contagiosa is very prone to attack flexures of the body, particularly those behind and below the ears, at the angles of the nose, mouth, and eyes. When these areas are affected, fissuring usually takes place in the depth of the fold and these are often troublesome to heal. The secret of treatment consists in immobilising the areas surrounding the fissures. In the case of the ear, most of the trouble is kept up by the parent pulling out the ear to dress it. If the dressing is slipped in behind the ear, in the first instance using moist dressings such as 1 in 1000 flavine, or 1 in 4000 perchloride of mercury, and later using the mercury-Lassar's paste described above, healing usually takes place quickly.

More trouble is experienced when the angles of the mouth or eyes are affected. In the former case, it is often necessary to apply a piece of strapping across the angle of the mouth to procure healing, while in the htter case the eye may be firmly bandaged up.

\section{Eczematised Impetigo.}

In some cases, especially in underfed and unhealthy children, a condition which begins as an impetigo may gradually be converted into a raw, red weeping area, which fails to respond to the ordinary treatment used for impetigo. This condition is often seen around the ears of children who have a purulent ear discharge. It frequently invades the scalp, and here a further complication occurs in the form of a pustular folliculitis. This so-called "pustular eczema of the scalp" is one of the most troublesome skin conditions which we meet with in children. Owing to the acutely sensitive state of the skin, it is usually impossible to apply antiseptics. Starch poultices with 1 in 1000 flavine may be applied for half an hour from time to time to remove crusts, but the most suitable applications in the early stages are astringent lotions, such as aluminium acetate or lead lotions. These should be changed frequently and their use continued till the congestion and weeping have ceased. After this a scaly stage supervenes in which very weak (1 per cent.) sulphur and salicylic acid ointments may be tolerated, but it may be necessary to return to the lotions from time to time. Various other remedies may have to be tried in some cases, but it is impossible to say more about them now.

\section{Pityriasiform Streptococcal Dermatitis.}

Low-grade infections with streptococci sometimes give rise to patches of chronic scaly dermatitis, indistinguishable as regards the lesions themselves, from patches of scaly dermatitis due to other irritants. One can often see typical impetigo lesions becoming merged into these fairly welldefined patches. One of the best known forms of this type of streptococcal infection is seen in the condition known as pityriasis alba. Here we have one or more sharply defined, pale pinkish patches covered by fine branny scales and have a crêpe-like surface, commonly seen on the faces of young children. These patches are not always due to streptococcal infection; frequently they are produced by dribbling or by improper drying of the face, but in some cases streptococci can be isolated and the infection may be spread to other children. Such patches usually respond to the following ointment :-

$\begin{array}{lllll}\text { R. Ac. salicyl.. } & \ldots & \ldots & \ldots & \text { gr. xv. } \\ \text { Hydrarg. perchlor. } \ldots & \ldots & \text { gr. } \frac{1}{8} \\ \text { Cerat. galeni (B.P.C.) } & \ldots & \text { ad } 3 i .\end{array}$

\section{Ecthyma.}

Here we have a condition which somewhat simulates impetigo contagiosa. The lesions do not often appear on the face, however, but are most common on the extremities, particularly about the knees. The lesions differ from impetigo in the fact that the crust is not "stuck on" the surface, but is, as it were, embedded in the skin, its surface being flush with the skin, and is surrounded by a well-defined red inflammatory zone. When the crust is removed a definite ulcer is seen and this, when healed, tends to leave a scar. These lesions are seen chiefly in itchy conditions, such as scabies, pediculosis, or urticaria papulosa, and are undoubtedly due to a combination of two factors, scratching and septic infection. In the itchy dermatosis scratching is the primary factor and infection follows when the surface epithelium has been excoriated. In a few cases, however, the condition may commence as a simple impetigo and the scratching may be an added factor, thus the term " ulcerating impetigo" sometimes applied to this condition is only applicable to certain cases. Staphylococcal lesions, folliculitis, and boils may also in some instances precede ecthymatous lesions.

The treatment consists first in removing, as far as possible, the cause of the itching. This being done, the lesions should be treated much on the lines of an impetigo, but, after removal of crusts, the mercury-Lassar's paste should be spread on linen and tied on, being changed twice or thrice daily according to the amount of exudate. 


\section{Staphylococcal Infections.}

I have now discussed the main affections of the skin due to streptococcal infection; it remains to say a few words about staphylococcal infections. Although the Staphylococcus epidermidis albus is a constant inhabitant of the skin, pure infections by pyogenic staphylococci are not so common as is the case with pyogenic streptococci. It is probable, however, that most of the septic complications of ordinary moist dermatoses are due to staphylococci, but owing to the varied flora found under these conditions, it is difficult to apportion blame.

The following are the commonest staphylococcal lesions found in children :-

\section{Boclihart's Impetigo.}

This is a superficial pustulation of the hair follicles, a folliculitis, which is not very common by itself, but sometimes complicates septic dermatitis of the hairy regions. It is best seen in the condition called "pustular eczema of the scalp" already referred to. The pustules are very superficial and do not extend down the hair follicles to any depth; they usually give pure cultures of staph. pyogenes aureus. They are resistant to treatment and should not, as a rule, be treated with any ointment or greasy preparation. Spirit lotions with, weak antiseptics are most successful, such as 1 in 4000 perchloride of mercury in 60 per cent. alcohol, or liq. calcis sulphurat. $3 \mathrm{i}$., sp. vin. rect. ろii., glycerin 3ii., aq. ad ऊ viii. When much general dermatitis is present, lead or aluminium acetate lotion is better tolerated.

Vaccines are worth a trial in resistant cases, but are only occasionally helpful. Epilation by means of $X$ rays is necessary in some cases.

\section{Furunculosis.}

Boils in children are often complications of other infected skin conditions, and usually subside when the primary condition is treated. It is necessary, however, to treat each boil with proper surgical precautions, so as to prevent spread of infection. I am not personally in favour of early incision, but as soon as pus points, the boil should be pricked and the pus, usually quite a small amount, gently evacuated. Boric fomentations should never be applied as they tend to spread infection. Compresses of 1 in 4000 perchloride of mercury are the best dressings. No further treatment is necessary, unless further crops of boils develop. This is an indication that the patient has become hypersensitive to staphylococci, and general treatment should be adopted to try to raise the patient's resistance. If vaccines are given, they should be used with great caution and very small doses tried in the first instance, or the condition may become worse. General tonics are useful and sulphur may also be given internally, either as colloidal sulphur or calcium sulphide. Injections of colloidal manganese have proved useful in some casesc Boils in children, however, are rarely as trouble some as in adults, and treatment on general line $\$$ is usually quickly successful.

\section{Multiple Abscesses in Infants.}

Some debilitated infants develop crops of subs cutaneous abscesses, usually painless, which 理 cultured grow Staph. pyogenes aureus. These are apt to end fatally, unless dealt with in the earlo stages. The treatment is mainly surgical, evacuae tion of the pus and careful dressings being th\& important thing. The infant should be kept warm and put on a suitable diet, so as to increase its body-weight.

These are the commoner affections due to pyogenig organisms met with in infants and children; othes secondary infections can be dealt with on simila lines.

\section{BROMIDE ERUPTIONS.}

\section{BY}

H. HALDIN-DAVIS, M.D. Oxf., F.R.C.S. Excre DERMATOLOGIST TO THE ROYAL FREE HOSPITAL.

THese eruptions are of course well knowrg especially to physicians who are concerned wit the treatment of nervous diseases, above all wit? epilepsy. Their commonest manifestations ar: illustrated well by the name often employed to describe the appearances produced-i.e., "bromid acne," which emphasises the point that the salien characteristic of all cutaneous bromodermia is the production of pus. But there are several variac tions from the common type. In many cases the lesions are extensive and take on a somewh warty appearance, with pus exuding from a numbè of tiny openings. Such cases usually offer particular difficulty in diagnosis as they show then selves under circumstances in which bromodermi is expected, although they are of practical impog tance as they interfere with the treatment of thes nervous condition for which the bromide is bein administered and may even cause its interruption Such cases are described as being of the " mycotic" or frambœsiform type of bromodermia, and a goode discussion of this type of eruption will be found in the May number of the British Journal of Dermatology, 1923, by Parkes Weber, who had some patients very severely affected under his carø, patients, in fact, in whom ulceration and granules matous tumour formation were so conspicuous that unless their condition had always varied exact $\vec{Q}$ with the exhibition or withdrawal of their medicin it would have been hard to believe that the bromide had caused the terrible cutaneous lesions fro which they suffered. A similar case was show by Sibley before the Dermatological Section of tige Royal Society of Medicine in 1917, and doubt 